\title{
Port-Hamiltonian based control for human-robot team interaction
}

\author{
Martin Angerer, Selma Musić and Sandra Hirche
}

\begin{abstract}
In this paper we consider a human commanding the overall behavior of a robot team while the robots are controlled to comply with formation constraints. Such humanrobot team interaction is challenging in terms of system complexity and control synthesis. The port-Hamiltonian framework is suitable for modeling the interconnected systems. We model the robotic team, cooperatively manipulating an object, as a constrained port-Hamiltonian system.

Furthermore, we propose a passivity-based control approach in the port-Hamiltonian framework for the cooperative manipulation system guided by the human. The control mechanism is based on the energy shaping for achieving a desired behavior of the formation and its preservation. An energy tank in the cascade is introduced to guarantee passivity of the complete system and safe interaction with humans in the robot environment. We validate the proposed approach with simulation and experiments.
\end{abstract}

\section{INTRODUCTION}

Human-robot interaction is a wide area of research with one of the main goals being to exploit the complementary capabilities of humans and robots. On the one side humans are inherently adaptable to uncertainties and have planning capabilities. On the other side robots are able to conduct tasks repetitively and with high precision. Furthermore, in a wide range of tasks it is necessary that humans and robots share the common workspace. Therefore, it is necessary to guarantee human safety during interaction. In human-robot team interaction the human typically knows the common goal and commands the team to achieve it, while the robots conduct other sub-tasks autonomously, e.g. maintaining formation [1] and collision avoidance [2]. An example for human-robot team interaction is teleoperation. The human guides the team of robotic manipulators, cooperatively manipulating an object.

The port-Hamiltonian framework is a powerful method for modelling complex and interconnected systems [3]. It is based on the interconnection of atomic structure elements (e.g. inertias, springs and dampers for mechanical systems) and on the known energy function of the system, the Hamiltonian. Teleoperation of coordinated robot teams was studied in the port-Hamiltonian formulation [4]. Recently, physical human-robot team interaction in a cooperative manipulation set-up was modeled in the port-Hamiltonian framework [5]. However, modeling robot formation as a constrained portHamiltonian system is not done so far.

Control schemes for cooperative manipulation systems are

The work is partly supported by the European Union Seventh Framework Programme FP7/2007-2013 under grant agreement no. 601165 of the project "WEARHAP-Wearable Haptics for Humans and Robots".

The authors are with the Chair of Information-oriented Control, Technische Universität München (TUM), Munich, Germany typically object-centered and are based on impedance control and the grasp theory. Making impedance control physically interpretable motivated the development of the intrinsically passive control (IPC) approach within the port-Hamiltonian framework [6]. It is designed as an interconnection of virtual atomic structure elements to achieve a desired behavior in grasping. Virtual structures are commonly used in formation control to establish a desired geometric shape of robotic agents [7]. Therefore, it is reasonable to design a control strategy for robot formations within the port-Hamiltonian framework. Actively stabilizing a port-Hamiltonian system at a certain energy level is termed as energy shaping control [8]. If it is represented within the port-Hamiltonian framework, we obtain the control by interconnection of the port-Hamiltonian system [9]. Control by interconnection of constrained port-Hamiltonian systems is not analyzed in literature. Another important control approach in the portHamiltonian framework is energy transfer control, with the energy tanks as its most widely used concept [10]. Energy tanks allow to bound the energy supply to the system. Appropriately limiting the energy in the system enhances the safety in interaction with the environment and/or humans on-site. Approaches to maintain a safe level in single robots modify the given reference trajectory [11], or adapt the internal behaviour of the robot [12].

The first contribution in this paper is the modeling of a cooperative manipulation system as a constrained portHamiltonian system. The objective of the novel, passivitybased controller is two-fold: 1) to achieve a desired motion of the object, commanded by the human and 2) to achieve coordination between the manipulators. For that purpose we propose an interaction control mechanism represented in port-Hamiltonian form. It is a cascade of control by interconnection and energy transfer control laws. Exploiting the interconnection property of port-Hamiltonian systems by representing the controller within the framework has not been unified and practically considered. Additionally, considering the human safety in a shared workspace using the energy transfer control has not been done so far. In order to guarantee the passivity of the complete system, the energy tank ensures a limited energy supply and enhances the human's safety. We validate the approach by simulation and experiment.

The paper is structured as follows. In Section II the main theoretical concepts are outlined. In Section III the portHamiltonian model of the cooperative manipulation system is presented. In Section IV the control approach is proposed and analyzed. The simulation and experimental results are provided in the Section $\mathrm{V}$, and the conclusive remarks in 
Section VI.

\section{BACKGROUND}

In this section we briefly introduce the main theoretical concepts of the port-Hamiltonian framework.

\section{A. port-Hamiltonian framework}

The port-Hamiltonian framework is based on a known energy function, the Hamiltonian $(\mathcal{H})$, and provides the energy-consistent description of a physical system [3]. As the power conservative interconnection of port-Hamiltonian systems is again a port-Hamiltonian system, this framework is suitable for modeling complex and interconnected systems. The modeling of mechanical systems in the portHamiltonian framework can be achieved by interconnecting atomic structure elements: inertias, dampers and springs. Every atomic element is defined by a Hamiltonian energy function and interacts with the other elements by exchanging energy through a port. The port is described by a pair of variables (e.f) representing efforts and flows, respectively. Their dual product is power.

An input-state-output form of a port-Hamiltonian system with the Hamiltonian function $\mathcal{H}$ is:

$$
\begin{aligned}
\dot{\boldsymbol{x}} & =[J(\boldsymbol{x})-D(\boldsymbol{x})] \frac{\partial \mathcal{H}}{\partial \boldsymbol{x}}(\boldsymbol{x})+G(\boldsymbol{x}) \boldsymbol{u} \\
\boldsymbol{y} & =G^{T}(\boldsymbol{x}) \frac{\partial \mathcal{H}}{\partial \boldsymbol{x}}(\boldsymbol{x}),
\end{aligned}
$$

where $J(\boldsymbol{x})$ is a skew-symmetric structure matrix and $D(\boldsymbol{x}) \succeq 0$ is a symmetric dissipation matrix. Inputs to the system are flows, $\boldsymbol{u}$, and outputs are efforts $\boldsymbol{y}$. The matrix $G(\boldsymbol{x})$ is a mapping matrix and $\boldsymbol{x}$ is the state vector of the system. The Hamiltonian of the system is a positive, semidefinite function $\mathcal{H} \geq 0$, which represents the total energy stored in the system. The rate of the energy change of (1) is:

$$
\dot{\mathcal{H}}=\boldsymbol{y}^{T} \boldsymbol{u}-\frac{\partial^{T} \mathcal{H}}{\partial \boldsymbol{x}} D(\boldsymbol{x}) \frac{\partial \mathcal{H}}{\partial \boldsymbol{x}}
$$

According to (2) every port-Hamiltonian system described in the form (1) is passive.

\section{B. Twists and wrenches}

We use coordinate frames associated with a body, i.e. noninertial frames. A transformation from the inertial frame, $\Psi_{0}$, to a frame $\Psi_{i}$ is given by a homogeneous matrix:

$$
H_{0}^{i}:=\left(\begin{array}{ll}
R_{0}^{i} & p_{0}^{i} \\
\mathbf{0}_{1 \times 3} & 1
\end{array}\right) \in S E(3)
$$

which consists of a translation vector $\boldsymbol{p}_{0}^{i} \in \mathbb{R}^{3}$ and a rotation matrix $R_{0}^{i} \in S O(3)$.

In this paper we represent the rigid body motion with the twist notation (see e.g. [13]). The twist associated with the frame $\Psi_{i}$, relative to the frame $\Psi_{j}$ and expressed in the inertial frame $\Psi_{0}$ is:

$$
T_{i}^{0, j}=\left(\left(\boldsymbol{\omega}_{i}^{0, j}\right)^{T} \quad\left(\boldsymbol{v}_{i}^{0, j}\right)^{T}\right)^{T}
$$

and consists of an angular velocity $\boldsymbol{\omega}_{i}^{0, j} \in \mathbb{R}^{3}$ around a screw axis and a translational velocity $\boldsymbol{v}_{i}^{0, j} \in \mathbb{R}^{3}$ along the same axis. A coordinate transformation of a twist from $\Psi_{0}$ to $\Psi_{j}$ is given by

$$
T_{i}^{j, j}=T_{i}^{j}=A d_{H_{0}^{j}} T_{i}^{0, j}=\left(\begin{array}{cc}
R_{0}^{j} & 0 \\
\tilde{p}_{0}^{j} R_{0}^{j} & R_{0}^{j}
\end{array}\right) T_{i}^{0, j},
$$

where $\tilde{p}_{0}^{j}$ is the skew-symmetric matrix of $\boldsymbol{p}_{0}^{j}$. The dual quantity of a twist is a wrench. The wrench $W_{i}^{0}$ which acts on a body associated with the frame $\Psi_{i}$, expressed in the inertial frame $\Psi_{0}$ is:

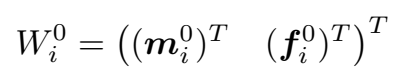

where $\boldsymbol{m}_{i}^{0} \in \mathbb{R}^{3}$ are moments and $\boldsymbol{f}_{i}^{0} \in \mathbb{R}^{3}$ are forces. A coordinate transformation of a wrench from $\Psi_{0}$ to $\Psi_{j}$ is defined as:

$$
W_{i}^{j}=A d_{H_{j}^{0}}^{T} W_{i}^{0}
$$

\section{C. port-Hamiltonian formulation of mechanical elements}

Let us define the atomic mechanical elements in the portHamiltonian form.

1) Springs: A spring is defined between two bodies, $B_{i}$ and $B_{j}$, with coordinate frames $\Psi_{i}$ and $\Psi_{j}$ fixed to the bodies, respectively. The potential energy stored in the spring is a positive definite function of the displacement $H_{i}^{j}$ :

$$
\mathcal{H}_{\mathrm{P}}: S E(3) \rightarrow \mathbb{R}^{+} ; H_{i}^{j} \mapsto \mathcal{H}_{\mathrm{P}}\left(H_{i}^{j}\right) .
$$

Energy functions of different types of springs are summarized in [13]. The input-state-output form of the spring is defined by the displacement $H_{i}^{j}$ (state), the wrench $W_{i}^{i}$ (output) and the twist $T_{i}^{i, j}$ (input):

$$
\begin{aligned}
\dot{H}_{i}^{j} & =H_{i}^{j} T_{i}^{i, j} \\
W_{i}^{i} & =H_{i}^{j^{T}} \frac{\partial \mathcal{H}_{\mathrm{P}}\left(H_{i}^{j}\right)}{\partial H_{i}^{j}} .
\end{aligned}
$$

2) Inertias: The kinetic energy stored in a body is a function of its relative motion w.r.t. to an inertial reference frame:

$$
\mathcal{H}_{\mathrm{K}}\left(P_{b}^{b}\right)=\frac{1}{2}\left(P_{b}^{b}\right)^{T} M_{b}^{-1} P_{b}^{b}
$$

where $P_{b}^{b}$ is the momentum of a body $b$ expressed in a bodyfixed frame $\Psi_{b}$ and $M_{b}$ is the inertial matrix of the body. The input-state-output form of the body is defined by the momentum $P_{b}^{b}$ (state), the twist $W_{i}^{j}$ (output) and the twist $T_{i}^{j}$ (input):

$$
\begin{aligned}
\dot{P}_{b}^{b} & =C_{b} \frac{\partial \mathcal{H}_{\mathrm{K}}\left(P_{b}^{b}\right)}{\partial P_{b}^{b}}+I_{6} W_{b}^{b} \\
T_{b}^{b, 0} & =I_{6} \frac{\partial \mathcal{H}_{\mathrm{K}}\left(P_{b}^{b}\right)}{\partial P_{b}^{b}}
\end{aligned}
$$

where $C_{b}$ represents Coriolis and centrifugal forces, $I_{6}$ is the identity matrix of order 6 . 
3) Dampers: Energy dissipation is represented as an input-output mapping between wrenches and twists:

$$
W_{b}^{b}=F\left(T_{b}^{b, 0}\right) \text {. }
$$

For linear dampers, $D$, the wrench is directly proportional to the twist:

$$
W_{b}^{b}=D T_{b}^{b, 0}
$$

The dissipated (co-)energy in this case is:

$$
\mathcal{H}_{\mathrm{D}}^{*}=\frac{1}{2} T_{b}^{b, 0}{ }^{T} D T_{b}^{b, 0}
$$

\section{PORT-HAMILTONIAN MODELLING OF ROBOT TEAMS}

In this section we propose a port-Hamiltonian based model of a cooperative manipulation system. We represent it as a constrained model, in an implicit input-state-output form and derive the explicit input-state-output model. Let us assume a setting depicted in fig. (1) where $N$ manipulators are connected to the common object. The inertial frame is denoted as $\Psi_{0}$, the object frame as $\Psi_{b}$ and the end-effector frames as $\Psi_{i}$ where $i=1, \ldots, N$. The object and the end-effectors are represented as inertial elements, where the momentum of the object is $P_{b}^{b}$ and the momenta of the robots are $P_{b_{i}}^{b_{i}}$, $i=1, \ldots, N$. The potential energy of the complete system is represented as a single spring, connecting the object and the ground, with the displacement denoted as $H_{b}^{0}$.

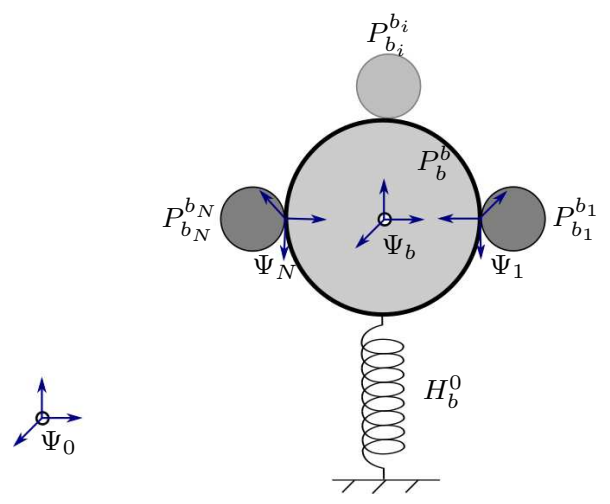

Fig. 1. Cooperative system model represented as a set of interconnected energy storing elements, inertias and a spring.

\section{A. Cooperative system modelling}

Let us represent the system depicted in fig. (1) in an implicit port-Hamiltoninan form:

$$
\begin{aligned}
\dot{\boldsymbol{x}} & =J(\boldsymbol{x}) \frac{\partial \mathcal{H}_{s}}{\partial \boldsymbol{x}}(\boldsymbol{x})+A(\boldsymbol{x}) \boldsymbol{\lambda}+G(\boldsymbol{x}) \boldsymbol{u}, \\
0 & =A^{T}(\boldsymbol{x}) \frac{\partial \mathcal{H}_{s}}{\partial \boldsymbol{x}}(\boldsymbol{x}), \\
\boldsymbol{y} & =G^{T}(\boldsymbol{x}) \frac{\partial \mathcal{H}_{s}}{\partial \boldsymbol{x}}(\partial x)
\end{aligned}
$$

where $\boldsymbol{x} \in \mathcal{M}$ is an $n$-dimensional state vector defined on a manifold, $J(\boldsymbol{x})$ is a skew-symmetric structure matrix, $A(\boldsymbol{x})$ is a constraint matrix with $\lambda \in \mathbb{R}^{l}$ being the constraining wrenches, $G(\boldsymbol{x})$ is the input mapping matrix, $\boldsymbol{u} \in \mathcal{F}$ an $m$ dimensional input vector, $\boldsymbol{y} \in \mathcal{F}^{*}$ an $m$-dimensional output vector and $\mathcal{H}_{s}$ is a Hamiltonian function of the model.
The state $\boldsymbol{x}$ in eq. (15) is a stacked vector of the configuration variable of the gravity spring and the momenta of the object and the $N$ manipulators: $\boldsymbol{x}^{T}=$ $\left[H_{b}^{0^{T}}, P_{b}^{b^{T}}, P_{b_{1}}^{b_{1} T}, \ldots, P_{b_{i}}^{b_{i} T}, \ldots, P_{b_{N}}^{b_{N}{ }^{T}}\right]^{T}$. The structure and the mapping matrices are

$$
\begin{aligned}
J(\boldsymbol{x}) & =\left(\begin{array}{ccccc}
0 & H_{b}^{0} & 0 & \cdots & 0 \\
-H_{b}^{0^{T}} & C_{b} & 0 & \cdots & 0 \\
0 & 0 & C_{b_{1}} & \cdots & 0 \\
\vdots & \vdots & \vdots & \ddots & \vdots \\
0 & 0 & 0 & \cdots & C_{b_{N}}
\end{array}\right), \\
G(\boldsymbol{x}) & =\left(\begin{array}{cccc}
0 & 0 & \cdots & 0 \\
A d_{H_{0}^{b}} & 0 & \cdots & 0 \\
0 & A d_{H_{0}^{b_{1}}} & \cdots & 0 \\
\vdots & \vdots & \ddots & \vdots \\
0 & 0 & \cdots & A d_{H_{0}^{b_{N}}}
\end{array}\right)
\end{aligned}
$$

where $C_{*}$ accounts for the Coriolis and centrifugal terms. The stacked input vector consists of the external wrenches acting on the inertias $\boldsymbol{u}=\left[W_{b}^{0}, W_{b_{1}}^{0}, \ldots, W_{b_{i}}^{0}, \ldots, W_{b_{N}}^{0}\right]$, expressed in the body-fixed frames. The outputs are the twists of the inertias $\boldsymbol{y}^{T}=\left[T_{b}^{0^{T}}, T_{b_{1}}^{0^{T}}, \ldots, T_{b_{i}}^{0^{T}}, \ldots, T_{b_{N}}^{0^{T}}\right]^{T}$. The Hamiltonian energy $\mathcal{H}_{s}$ is the sum of the energies of all the elements:

$$
\mathcal{H}_{s}(\boldsymbol{x})=\mathcal{H}_{\mathrm{P}}\left(H_{b}^{0}\right)+\mathcal{H}_{\mathrm{K}}\left(P_{b}^{b}\right)+\sum_{i=1}^{N} \mathcal{H}_{\mathrm{K}}\left(P_{b_{i}}^{b_{i}}\right) .
$$

The interaction of the manipulators with the rigid object imposes kinematic constraints on the complete system. This implies that there is no relative motion between the object and the end-effectors which can be denoted as:

$$
T_{b}^{0}=T_{b_{i}}^{0}, \forall i=1 . . N
$$

where $T_{b}^{0}$ denotes the twist of the virtual object and $T_{b_{i}}^{0}$ stands for the twist of the $i$ th manipulator. With a change of coordinates $T_{b}^{b, 0}=A d_{H_{b_{i}}^{b}} T_{b_{i}}^{b_{i}, 0}$ the constraint equation is:

$$
0=\underbrace{\left(\begin{array}{ccrrc}
0 & I_{6} & -A d_{H_{b_{1}}^{b}} & \cdots & 0 \\
\vdots & \vdots & \vdots & \ddots & \vdots \\
0 & I_{6} & 0 & \cdots & -A d_{H_{b_{N}}^{b}}
\end{array}\right)}_{A^{T}(\boldsymbol{x})} \frac{\partial \mathcal{H}_{s}}{\partial \boldsymbol{x}}(\boldsymbol{x})
$$

where $A(\boldsymbol{x}) \in \mathbb{R}^{n \times l}$ is the constraint matrix with $n$ being the number of states of the system and $l$ the number of independent kinematic constraints. The violation of constraints generates internal stress on the object [14]. We consider the constraining forces using Lagrangian multipliers $\boldsymbol{\lambda}$. Therefore, the port-Hamiltonian formulation of the constrained model is a set of differential and algebraic equations. To restore the explicit input-state-output form (1), the implicit model needs to be restricted to the constrained manifold $\mathcal{M}$ which can be achieved by eliminating the Lagrangian multipliers from eq. (15). 


\section{B. Elimination of constraints}

The differential-algebraic equation (15) can be reduced to a set of ordinary differential equations by multiplying it with a full-rank left annihilator of the constraint matrix, $A^{\perp}(\boldsymbol{x})$, such that $A^{\perp}(\boldsymbol{x}) A(\boldsymbol{x}) \boldsymbol{\lambda}=0$. The annihilator can be calculated as the kernel of $A(x)$. We use the left annihilator of the following form:

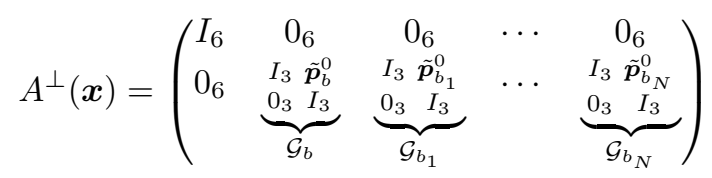

where the combined matrix $\mathcal{G}=\left[\mathcal{G}_{b}, \mathcal{G}_{b_{1}}, \ldots, \mathcal{G}_{b_{N}}\right] \in$ $\mathbb{R}^{6 \times 6(N+1)}$ is the grasp matrix. Multiplying eq. (15) with the annihilator (20) the following is obtained:

$$
A^{\perp} \dot{\boldsymbol{x}}=A^{\perp} J(\boldsymbol{x}) \frac{\partial \mathcal{H}_{s}}{\partial \boldsymbol{x}}(\boldsymbol{x})+A^{\perp} G(\boldsymbol{x}) \boldsymbol{u}
$$

Effectively, $A^{\perp} \dot{\boldsymbol{x}}$ is a coordinate transformation [15]. With the proposed annihilator of the constraint matrix, $A^{\perp}$, the configuration $H_{b}^{0}$ is unaffected by the transformation. For the momenta a new variable $\hat{P}_{b}^{b}=\mathcal{G}\left[P_{b}^{b^{T}}, P_{b_{i}}^{b_{i}{ }^{T}}\right]^{T}$ is introduced. The resulting state $\hat{\boldsymbol{x}}=\left[H_{b}^{0^{T}}, \hat{P}_{b}^{b T}\right]^{T}$ evolves on a constrained manifold:

$$
\mathcal{M}_{c}=\left\{\boldsymbol{x} \in \mathcal{M} \mid A^{T}(\boldsymbol{x}) \frac{\partial \mathcal{H}_{s}}{\partial \boldsymbol{x}}=0\right\} .
$$

The dimension of the state vector is reduced in the constrained space, $\hat{\boldsymbol{x}} \in \mathbb{R}^{n-6 N}$. The partial derivatives of the Hamiltonian function are modified to $\frac{\partial \mathcal{H}_{s}}{\partial \boldsymbol{x}}=A^{\perp^{T}} \frac{\partial \mathcal{H}_{s}}{\partial \hat{\boldsymbol{x}}}$. Now it is possible to obtain the model in an explicit input-stateoutput form:

$$
\begin{aligned}
\dot{\hat{\boldsymbol{x}}} & =\hat{J}(\boldsymbol{x}) \frac{\partial \hat{\mathcal{H}}_{s}}{\partial \hat{\boldsymbol{x}}}+\hat{G}(\boldsymbol{x}) \boldsymbol{u} \\
\boldsymbol{y} & =\hat{G}^{T}(\boldsymbol{x}) \frac{\partial \hat{\mathcal{H}}_{s}}{\partial \hat{\boldsymbol{x}}}
\end{aligned}
$$

where $\hat{\mathcal{H}}_{s}$ is the Hamiltonian function evolving on the constrained manifold (22). The reduced structure matrix $\hat{J}(\boldsymbol{x})=A^{\perp} J(\boldsymbol{x}) A^{\perp^{T}}$ is again skew-symmetric, i.e. we obtain the explicit input-state-output port-Hamiltonian representation of the constrained system. The new mapping matrix $\tilde{G}(x)=\left[\begin{array}{ll}\tilde{G}_{b} & \tilde{G}_{\mathrm{m}}\end{array}\right]$ decomposes into two parts: $\tilde{G}_{b} \in \mathbb{R}^{12 \times 6}$ represents the interaction of the object with the environment. $\tilde{G}_{\mathrm{m}} \in \mathbb{R}^{12 \times 6 N}$ represents the interaction with the controller. The energy balance of (23) is $\dot{\hat{\mathcal{H}}}_{s}=\boldsymbol{y}^{T} \boldsymbol{u}$, i.e. the system is power-conservative.

\section{CONTROl STRATEgy FOR HUMAN-ROBOt TEAM INTERACTION}

In this section we propose a passivity-based control methodology within the port-Hamiltonian framework for the human-robot team interaction in which the human commands the overall behavior of the team, while the robot team manipulates and maintains the grasp of the object. The control approach is a cascade of energy shaping control strategy with the damping injection and the energy transfer control.

Energy shaping is achieved by exploiting the available information on the energetic state obtained from the controller and the modeled system. A maximum level of the energy to be stored in the controller and the system limits both the velocity and the forces. The energy bound of the controllersystem interconnection is ensured by sourcing it from an energy tank. The block structure of the complete system is depicted in fig. (2).

\section{A. Energy shaping and damping injection for cooperative manipulation}

The controller we propose has a physical interpretation since it is based on the virtual mechanical structures assumed to interconnect the robots and the human with the object. Therefore, the controller can be represented in the portHamiltonian framework. There are two alternatives to establish a desired formation of robots around the held object: 1) the use of non-zero rest-length springs connecting the robots pairwise, or 2) the introduction of a virtual object as a hinge point [16]. We use the virtual object concept. The virtual object is connected by a virtual spring and a virtual damper to the human hand. In subsection IV-D we introduce variable stiffness and damping parameters to shape the energy flow towards the controller, affected by the human commands. The end-effectors are coupled to the surface of the virtual object with constant virtual springs and virtual dampers. The proposed virtual structure is depicted in fig. (3).

Due to space constraints only an interconnection of the $i$ th manipulator is given in the equations of the controller. Their extension to $N$ interconnections is straightforward. We represent the controller in the port-Hamiltonian framework as:

$$
\begin{aligned}
\dot{\boldsymbol{x}}_{c} & =\left[J_{c}\left(\boldsymbol{x}_{c}\right)-D_{c}\left(\boldsymbol{x}_{c}\right)\right] \frac{\partial \mathcal{H}_{c}}{\partial \boldsymbol{x}_{c}}\left(\boldsymbol{x}_{c}\right)+G_{c}\left(\boldsymbol{x}_{c}\right) \boldsymbol{u}_{c} \\
\boldsymbol{y}_{c} & =G_{c}^{T}\left(\boldsymbol{x}_{c}\right) \frac{\partial \mathcal{H}_{c}}{\partial \boldsymbol{x}_{c}}\left(\boldsymbol{x}_{c}\right)
\end{aligned}
$$

with the components:

$$
\begin{aligned}
\dot{\boldsymbol{x}}_{c} & =\left(\begin{array}{c}
\dot{H}_{v}^{d} \\
\dot{P}_{v}^{v} \\
\dot{H}_{v}^{i}
\end{array}\right), \frac{\partial \mathcal{H}_{c}}{\partial \boldsymbol{x}_{c}}=\left(\begin{array}{c}
\frac{\partial \mathcal{H}_{c}}{\partial H^{d}} \\
\frac{\partial \mathcal{H}_{c}}{\partial P_{v}^{v}} \\
\frac{\partial \mathcal{H}_{c}}{\partial H_{v}^{i}}
\end{array}\right), \boldsymbol{u}_{c}=\left(\begin{array}{c}
T_{d}^{0} \\
T_{i}^{0}
\end{array}\right), \boldsymbol{y}_{c}=\left(\begin{array}{l}
W_{d}^{0} \\
W_{i}^{0}
\end{array}\right) \\
J_{c} & =\left(\begin{array}{ccc}
0 & H_{v}^{d} & 0 \\
-H_{v}^{d^{T}} & C_{v} & -H_{v}^{i T} \\
0 & H_{v}^{i} & 0
\end{array}\right), \\
D_{c} & =\left(\begin{array}{ccc}
0 & 0 & 0 \\
0 & D_{v}+D_{i}+D_{0} & 0 \\
0 & 0 & 0
\end{array}\right), \\
G_{c} & =\left(\begin{array}{lll}
G_{c_{1}} & G_{c_{2}}
\end{array}\right)=\left(\begin{array}{cc}
-H_{v}^{d} A d_{H_{0}^{v}} & 0 \\
D_{v} A d_{H_{0}^{v}} & D_{i} A d_{H_{0}^{v}} \\
0 & -H_{v}^{i} A d_{H_{0}^{v}}
\end{array}\right)
\end{aligned}
$$

. The state vector $\boldsymbol{x}_{c}$ is a stacked vector of:

1) the variable virtual spring, $H_{v}^{d}$, connecting the human hand and the virtual object,

2) the virtual object $v$ momenta, $P_{v}^{v}$, and 


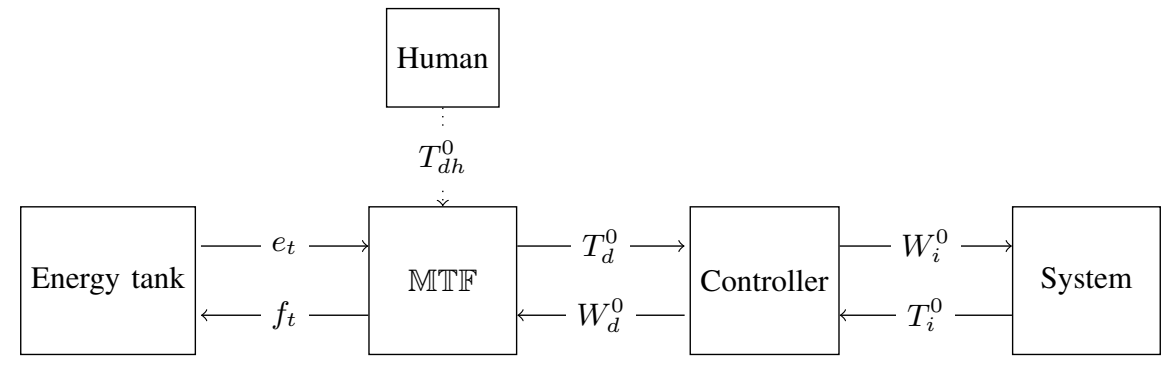

Fig. 2. Block structure of the complete system: The Energy tank supplies energy to the Controller, while the human is energetically decoupled (indicated by dotted lines). The energy flow depends on the human input and is controlled via a Modulated Transformer (MTIF). The output of the transformer is the (modified) reference trajectory $T_{d}^{0}$. The wrench applied by the end-effectors is $W_{i}^{0}$ and the twists of the end-effectors are $T_{i}^{0}$.

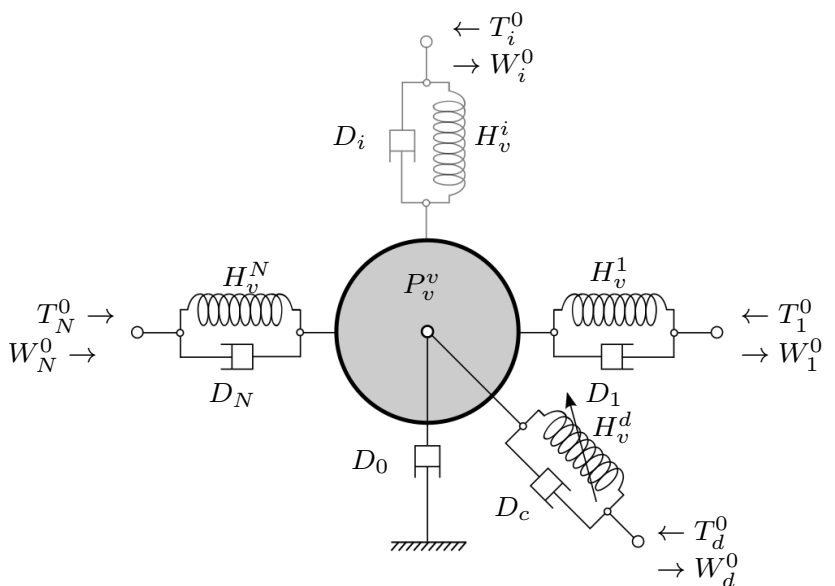

Fig. 3. Interaction controller represented as a virtual structure of inertia (variable) springs and (variable) dampers.

3) the virtual spring, $H_{v}^{i}$, connecting the virtual object and the $i$-th end-effector.

The Hamiltonian energy of the controller is the sum of the energies stored in all its atomic elements:

$$
\mathcal{H}_{c}\left(\boldsymbol{x}_{c}\right)=\mathcal{H}_{\mathrm{P}}\left(H_{v}^{d}\right)+\sum_{i=1}^{N} \mathcal{H}_{\mathrm{P}}\left(H_{v}^{i}\right)+\mathcal{H}_{\mathrm{K}}\left(P_{v}^{v}\right) .
$$

The controller is energy-conservative and due to the assigned damping it is also passive. It is suitable for achieving both the desired velocity set-point and formation preservation of the robots. The formation preservation ensures that the constraints are not violated. In the next subsection the interconnection of the model and the controller is studied.

\section{B. Connection of the controller and the model}

The output of the controller, $W_{i}^{0}$, is the input for the model and the output of the model, $T_{i}^{0}$, is the input for the controller. Therefore, the interconnected system is:

$$
\begin{aligned}
& \underbrace{\left(\begin{array}{c}
\dot{\hat{\boldsymbol{x}}} \\
\dot{\boldsymbol{x}}_{c}
\end{array}\right)}_{\dot{\boldsymbol{x}}_{c s}}=\underbrace{\left(\begin{array}{cc}
\hat{J} & \hat{G}_{\mathrm{m}} G_{c 2}^{T} \\
-G_{c 2} \hat{G}_{\mathrm{m}}^{T} & J_{c}-D_{c}
\end{array}\right)}_{J_{c s}} \underbrace{\left(\begin{array}{c}
\frac{\partial \mathcal{H}_{c s}}{\partial \hat{\partial}} \\
\frac{\partial \mathcal{H}_{c s}}{\partial \boldsymbol{x}_{c}}
\end{array}\right)}_{\partial \mathcal{H}_{c s} / \partial \boldsymbol{x}_{c s}}+ \\
& +\underbrace{\left(\begin{array}{ll}
\hat{G}_{b} & G_{c 1}
\end{array}\right)}_{G_{c s}}\left(\begin{array}{c}
W_{b}^{0} \\
T_{d}^{0}
\end{array}\right) \\
& \left(\begin{array}{c}
T_{b}^{0} \\
W_{d}^{0}
\end{array}\right)=\left(\begin{array}{ll}
\hat{G}_{b}^{T} & G_{c 1}^{T}
\end{array}\right)\left(\begin{array}{c}
\frac{\partial \mathcal{H}_{c s}}{\partial \hat{x}} \\
\frac{\partial \hat{H}_{c s}}{\partial \boldsymbol{x}_{c}}
\end{array}\right)
\end{aligned}
$$

The external ports of the controller-model interconnection are for interaction with the human via the port $\left(T_{d}^{0}, W_{d}^{0}\right)$ and for interaction with the environment via the port $\left(W_{b}^{0}, T_{b}^{0}\right)$, i.e. via the object. The interconnection given with eq. (27) is passive as the interconnected subsystems are passive. In the next subsection we energetically decouple the human guiding the cooperative system to ensure passivity of the complete system and safety for humans on-site.

\section{Energy transfer control}

As the human and the system are not physically coupled, but the human commands the system with hand gestures, we interconnect them with virtual structures. In order to guarantee both stability and safety, we propose an energy transfer control in the form of an energy tank that supplies the controller and the system. The energy tank maintains a safe energy level in the system and can be integrated into the port-Hamiltonian representation of the complete, controlled system.

For human-robot interaction various safety metrics exist, see [17] for an overview. In this paper we limit the focus to the energy-based injury criteria. Experimental studies indicate minimal amounts of energy that cause a cranial bone failure [18] and a fracture of neck bones [19]. Based on these results, we define a maximum amount of energy that can be stored in the system. It represents the maximum level of the energy that can be stored in the tank:

$$
\mathcal{H}_{t}^{\max }= \begin{cases}517 \mathbf{J} & \text { adult cranium bone failure } \\ 30 \mathrm{~J} & \text { neck fracture }\end{cases}
$$

Let us formally analyze the combination of the energy tank and the controlled system, (27). The energy tank sources the controller and the controller resupplies its virtually dissipated energy to the energy tank. The energy tank is a virtual storage element defined with the Hamiltonian, $\mathcal{H}_{t}$. Let $x_{\mathrm{t}} \in \mathbb{R}$ denote the (scalar) energy state of the tank and let $\mathcal{H}_{t}\left(x_{\mathrm{t}}\right)=\frac{1}{2} x_{\mathrm{t}}^{2}$. The input-state-output, port-Hamiltonian representation of the energy tank is:

$$
\begin{aligned}
& \dot{x_{\mathrm{t}}}=f_{\mathrm{t}} \\
& e_{\mathrm{t}}=\frac{\partial \mathcal{H}_{t}\left(x_{\mathrm{t}}\right)}{\partial x_{\mathrm{t}}}\left(=x_{\mathrm{t}}\right)
\end{aligned}
$$

where $\left(f_{\mathrm{t}}, e_{\mathrm{t}}\right)$ is the flow-effort pair. The re-routing of the virtually dissipated energy into the tank is accomplished by 
choosing the tank input as:

$$
f_{\mathrm{t}}=\frac{1}{x_{\mathrm{t}}} \frac{\partial^{T} \mathcal{H}_{c s}}{\partial \boldsymbol{x}_{c s}} D_{c} \frac{\partial \mathcal{H}_{c s}}{\partial \boldsymbol{x}_{c s}}+\tilde{f}_{\mathrm{t}}
$$

where the first term of eq. (29) represents the dissipated power. In order to have an open port to connect the tank to the controller, we introduce a new input $\tilde{f}_{\mathrm{t}}$ to the energy tank. The energy balance of the tank is:

$$
\dot{\mathcal{H}}_{t}\left(x_{\mathrm{t}}\right)=e_{\mathrm{t}} f_{\mathrm{t}}=\frac{\partial^{T} \mathcal{H}_{c s}}{\partial \boldsymbol{x}_{c s}} D_{c} \frac{\partial \mathcal{H}_{c s}}{\partial \boldsymbol{x}_{c s}}+x_{\mathrm{t}} \tilde{f}_{\mathrm{t}}
$$

We propose to interconnect the tank and the controller by a modulated transformer $\mathbb{M T F}$ [20]. An $\mathbb{M T F}$ is a lossless element to dynamically shape the energy transfer by its variable transformer ratio $\boldsymbol{n}$. The transformer ratio determines the energy flow and is set by the human to specify the desired velocity $T_{d, h}^{0}$. The $\mathbb{M T F}$ representation in the portHamiltonian framework is:

$$
\begin{aligned}
T_{d}^{0} & =\boldsymbol{n} e_{\mathrm{t}} \\
\tilde{f}_{\mathrm{t}} & =-\boldsymbol{n}^{T} W_{d}^{0}
\end{aligned}
$$

where $\boldsymbol{n}$ is dynamically adapted to replicate the human command:

$$
\boldsymbol{n}=\frac{T_{d, h}^{0}}{x_{\mathrm{t}}}
$$

A combined port-Hamiltonian representation of the controlled system and the tank is as follows:

$$
\left(\begin{array}{c}
\dot{\boldsymbol{x}}_{c s} \\
\dot{x}_{\mathrm{t}}
\end{array}\right)=\left(\begin{array}{cc}
J_{c s} & \frac{T_{d}^{0}}{x_{\mathrm{t}}} \\
-\frac{T_{d}^{0 T}}{x_{\mathrm{t}}}+\frac{1}{x_{\mathrm{t}}} \frac{\partial^{T} \overline{\mathcal{H}}}{\partial \boldsymbol{x}_{c s}} D_{c} & 0
\end{array}\right)\left(\begin{array}{c}
\frac{\partial \overline{\mathcal{H}}}{\partial \boldsymbol{x}_{c s}} \\
\frac{\partial \mathcal{H}}{\partial x_{\mathrm{t}}}
\end{array}\right)
$$

The combined system is lossless for free object motion, i.e. $W_{b}^{0}=0$,

$$
\frac{d}{d t} \overline{\mathcal{H}}\left(\boldsymbol{x}_{c s}, x_{\mathrm{t}}\right)=\left(\begin{array}{ll}
\frac{\partial^{T} \overline{\mathcal{H}}}{\partial \boldsymbol{x}_{c s}} & \frac{\partial^{T} \overline{\mathcal{H}}}{\partial x_{\mathrm{t}}}
\end{array}\right)\left(\begin{array}{c}
\dot{\boldsymbol{x}}_{c s} \\
\dot{x}_{\mathrm{t}}
\end{array}\right)=0
$$

where the Hamiltonian $\overline{\mathcal{H}}\left(\boldsymbol{x}_{c s}, x_{\mathrm{t}}\right)$ is the total energy of tank, controller and system.

\section{Energy shaped stiffness and damping}

When $x_{t}=0$, the energy tank is depleted and unable to passively perform the action commanded by the human, $T_{d, h}^{0}$. A total depletion is avoided by suspending the input, i.e. $T_{d, h}^{0}=0$ if $x_{t}<\epsilon$, in a neighbourhood $\epsilon>0$ [20]. Discarding the input results in a loss of information and a permanent set-point deviation. We propose to avoid a depletion of the energy tank without modifying the human input, but by making the coupling of set-point and virtual object velocity variable. By adapting the coupling stiffness and damping as a function of the available energy, the flow of energy between tank and controller is shaped. Stiffness and damping are constant if the energy in the tank is sufficient for executing the velocity command. If the tank level falls below a certain threshold, the coupling is relaxed to limit the power flow. If the tank level is close to zero, $\mathcal{H}_{\mathrm{t}}(\mathrm{t})<\epsilon$, the energy transfer is completely disabled by a zero stiffness and damping.
A change of stiffness affects the energy stored in the spring. Relaxing stiffness sets energy free, increasing stiffness requires the supply of energy. Let us assume an a stiffness-dependent Hamiltonian for a spring

$$
\mathcal{H}_{\mathrm{P}}: S E(3) \times \mathcal{K} \rightarrow \mathbb{R}^{+} ;\left(H_{b}^{v}, \kappa\right) \mapsto \mathcal{H}_{\mathrm{P}}\left(H_{b}^{v}, \kappa\right),
$$

where $\kappa$ is the variable stiffness, a function of the tank level $\mathcal{H}_{t}$ : above a certain threshold $\mathcal{H}_{t}^{t h}$ the stiffness is constant. When the tank gets close to depletion the stiffness is reduced

$$
\kappa= \begin{cases}K_{d v} & \text { if } \mathcal{H}_{t}\left(x_{\mathrm{t}}\right) \geq \mathcal{H}_{t}^{t h} \\ K_{d v} \frac{\mathcal{H}_{t}\left(x_{\mathrm{t}}\right)-\epsilon}{\mathcal{H}_{t}^{t h}} & \text { if } \epsilon \leq \mathcal{H}_{t}\left(x_{\mathrm{t}}\right)<\mathcal{H}_{t}^{t h} \\ 0 & \text { if } \mathcal{H}_{t}\left(x_{\mathrm{t}}\right)<\epsilon\end{cases}
$$

where $K_{d v} \in \mathbb{R}^{6 \times 6}$ is a stiffness matrix. The rate of energy change w.r.t a variable stiffness $\kappa$ is

$$
\dot{\mathcal{H}}_{\kappa}=\frac{\partial \mathcal{H}_{\kappa}}{\partial \kappa} \frac{d \kappa}{d t}=e_{\kappa}^{T} f_{\kappa}
$$

which corresponds to the product of effort $\left(\frac{\partial \mathcal{H}_{\mathrm{P}}}{\partial \kappa}\right)$ and flow $(\dot{\kappa})$ and forms a power port $\left(f_{\kappa}, e_{\kappa}\right)$. The port-Hamiltonian representation of a variable stiffness spring is given by

$$
\begin{aligned}
\left(\begin{array}{c}
\dot{H}_{v}^{d} \\
\dot{\kappa}
\end{array}\right) & =\left(\begin{array}{cc}
H_{v}^{d} A d_{H_{0}^{v}} & 0 \\
0 & I
\end{array}\right)\left(\begin{array}{c}
T_{v}^{0}-T_{d}^{0} \\
f_{\mathrm{k}}
\end{array}\right) \\
\left(\begin{array}{c}
W_{v}^{0} \\
e_{\mathrm{k}}
\end{array}\right) & =\left(\begin{array}{cc}
A d_{H_{0}^{v}}^{T} H_{v}^{d^{T}} & 0 \\
0 & I
\end{array}\right)\left(\begin{array}{c}
\frac{\partial \mathcal{H}_{\kappa}}{\partial H_{v}^{d}} \\
\frac{\partial \mathcal{H}_{\kappa}}{\partial \kappa}
\end{array}\right) .
\end{aligned}
$$

One can express $\kappa$ as a function of the tank level using (30):

$$
\dot{\kappa}= \begin{cases}0 & \text { if } \mathcal{H}_{t}\left(x_{\mathrm{t}}\right) \geq \mathcal{H}_{t}^{t h} \\ \frac{K_{d v}}{\mathcal{H}_{t}^{t h}} \dot{\mathcal{H}}_{t}\left(x_{\mathrm{t}}\right)=\frac{K_{d v}}{\mathcal{H}_{t}^{t h}} \dot{x}_{\mathrm{t}} \frac{\partial \overline{\mathcal{H}}}{\partial x_{\mathrm{t}}} & \text { if } \mathcal{H}_{t}\left(x_{\mathrm{t}}\right)<\mathcal{H}_{t}^{t h}\end{cases}
$$

The power exchanged between the variable spring and the tank due to variability of the stiffness is:

$$
\dot{\overline{\mathcal{H}}}=\frac{\partial \overline{\mathcal{H}}}{\partial \kappa} \dot{\kappa}= \begin{cases}0 & \text { if } \mathcal{H}_{t}\left(x_{\mathrm{t}}\right) \geq \mathcal{H}_{t}^{t h} \\ \frac{\partial \overline{\mathcal{H}}}{\partial \kappa} \frac{K_{d v}}{\mathcal{H}_{t}^{t h}} \dot{x}_{\mathrm{t}} \frac{\partial \overline{\mathcal{H}}}{\partial x_{\mathrm{t}}} & \text { if } \mathcal{H}_{t}\left(x_{\mathrm{t}}\right)<\mathcal{H}_{t}^{t h}\end{cases}
$$

The power exchanged by the energy tank is of the form $\dot{\mathcal{H}}_{t}\left(x_{\mathrm{t}}\right)=\frac{\overline{\mathcal{H}}}{\partial x_{\mathrm{t}}} f_{\mathrm{t}}$, therefore $\frac{\partial \overline{\mathcal{H}}}{\partial \kappa} \frac{K_{d v}}{\mathcal{H}_{t}^{t h}} \dot{x}_{\mathrm{t}}$ is an input for the energy tank. One can now obtain the port-Hamiltonian representation of the variable stiffness spring and the energy tank:

$$
\begin{aligned}
\left(\begin{array}{c}
\dot{\boldsymbol{x}}_{c s} \\
\dot{x}_{\mathrm{t}} \\
\dot{\kappa}
\end{array}\right) & =\left[\left(\begin{array}{ccc}
J_{c s} & \frac{T_{d}^{0}}{x_{\mathrm{t}}} & 0 \\
-\frac{T_{d}^{0 T}}{x_{\mathrm{t}}} & 0 & -\frac{K_{d v}}{\mathcal{H}_{\mathrm{t}}^{\mathrm{h}}} \dot{x}_{\mathrm{t}} \\
0 & \frac{K_{d v}}{\mathcal{H}_{\mathrm{th}}} \dot{x}_{\mathrm{t}} & 0
\end{array}\right)\right. \\
& \left.-\left(\begin{array}{cccc}
0 & 0 & 0 \\
-\frac{1}{x_{\mathrm{t}}} \frac{\partial \overline{\mathcal{H}}^{T}}{\partial \boldsymbol{x}_{c s}} D_{c} & 0 & 0 \\
0 & 0 & 0
\end{array}\right)\right]\left(\begin{array}{c}
\frac{\partial \mathcal{\mathcal { H }}}{\partial \boldsymbol{x}_{c s}} \\
\frac{\partial \mathcal{H}}{\partial x_{t}} \\
\frac{\partial \mathcal{H}}{\partial \kappa}
\end{array}\right)
\end{aligned}
$$

The combination of such a system with a variable stiffness spring and a tank is lossless:

$$
\frac{d}{d t} \overline{\mathcal{H}}\left(\boldsymbol{x}_{c s}, x_{\mathrm{t}}, \kappa\right)=0
$$


The dissipative structure $D_{v}$ can be changed without compromising passivity as long as $D_{v} \succeq 0$. The mapping matrix $G_{c}$ in eq. (27) shows that the damping directly influences the energy exchange. We propose to reduce the damping in the same manner as for the stiffness. With a depletion of the energy tank the damping $D_{d v} \in \mathbb{R}^{6 \times 6}$ parallel to the variable spring is reduced. The coupling between the human and the virtual object is relaxed:

$$
\delta= \begin{cases}D_{d v} & \text { if } \mathcal{H}_{t}\left(x_{\mathrm{t}}\right) \geq \mathcal{H}_{t}^{t h} \\ D_{d v} \frac{\mathcal{H}_{t}\left(x_{\mathrm{t}}\right)}{\mathcal{H}_{t}^{t h}} & \text { if } \epsilon \leq \mathcal{H}_{t}\left(x_{\mathrm{t}}\right)<\mathcal{H}_{t}^{t h} \\ 0 & \text { if } \mathcal{H}_{t}\left(x_{\mathrm{t}}\right)<\epsilon\end{cases}
$$

\section{Results}

The model and the control strategy introduced in sections III and IV, respectively, are implemented in MAT$L A B /$ Simulink. The cooperative system of 2 robots with the masses $\mathbf{M}_{1,2}=10 \mathrm{~kg} \cdot I_{3}$ and the moments of inertia $\mathbf{J}_{1,2}=$ $0.5 \mathrm{kgm}^{2} \cdot I_{3}$ manipulates an object with the mass $\mathrm{M}_{o}=$ $1.4 \mathrm{~kg} \cdot I_{3}$ and the moment of inertia $\mathrm{J}_{o}=0.5 \mathrm{kgm}^{2} \cdot I_{3}$. The solver uses the Euler's method (odel) and the sample time is $1 \mathrm{~ms}$. The parameters of the controller are summarized in table I. Simulation results are shown in fig. (4). The available

TABLE I

CONTROLLER PARAMETERS

\begin{tabular}{l|l|l} 
& linear & angular \\
\hline Virt. inertia & $1.4 \mathrm{~kg} \cdot I_{3}$ & $0.5 \mathrm{kgm}^{2} \cdot I_{3}$ \\
\hline Damping & $250 \mathrm{~kg} / \mathrm{s} \cdot I_{3}$ & $30 \mathrm{kgm}^{2} / \mathrm{s} \cdot I_{3}$ \\
\hline Stiffness & $125 \mathrm{~N} / \mathrm{m} \cdot I_{3}$ & $15 \mathrm{Nm} \cdot I_{3}$
\end{tabular}

energy budget, $\mathcal{H}_{t}^{\max }=25 \mathrm{~J}$, complies with the safety limits from (IV-C). At $t=1 \mathrm{~s}$ the commanded velocity is too high w.r.t. the available energy budget, i.e. the energy at disposal is not sufficient to reach it. Therefore, the tank level is depleted. Trajectories exceeding the energetic limit are adapted with the variable stiffness and the damping in order to guarantee safety. The reduction of the stiffness and the damping starts as soon as the energy level falls under a threshold, $\mathcal{H}_{t}^{t h}=5 \mathrm{~J}$. As a consequence, the velocity is kept constant at the energetically feasible maximum until $t=2 \mathrm{~s}$ when the tank is refilled again and the commanded velocity is feasible. Limiting velocity and forces, the interaction safety of the system is enhanced. Additionally, the internal wrenches applied on the object are zero. The experiment is conducted with a robotic system of two KUKA LWR manipulators, mounted on a movable platform and depicted in the fig (5). The sampling time is $1 \mathrm{~ms}$. The obtained results are shown in fig. (6) and (7). In fig. (6) the tank energy level is depleted and the velocity is limited close to the maximum commanded velocity of $0.11 \mathrm{~m} / \mathrm{s}$. In fig. (7) the tank energy budget is enough to supply the system when the commanded velocity changes from $0.1-0.3[\mathrm{~m} / \mathrm{s}]$. The energy level does not reach the energetic threshold for the commanded velocity and is sufficient for reaching the desired velocity.

\section{CONCLUSION}

In this paper we developed a control scheme for humanrobot team interaction in a teleoperation setting for a co-

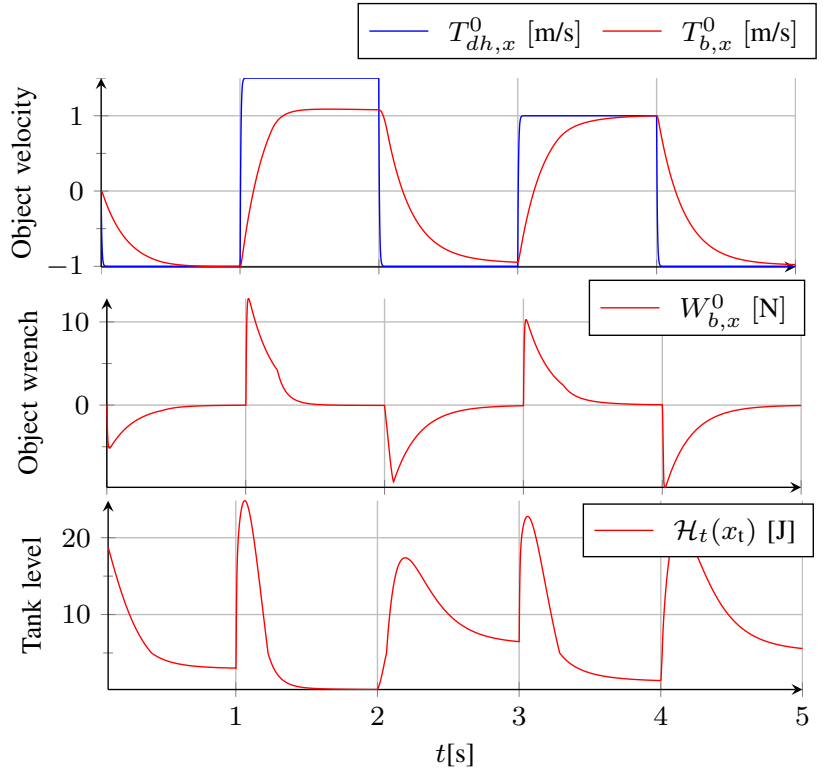

Fig. 4. Simulation results. Top: commanded and actual object velocity. Middle: force applied on the object. Bottom: energy level of the tank with $\mathcal{H}_{t}^{\max }=25 \mathrm{~J}$ and $\mathcal{H}_{t}^{t h}=5 \mathrm{~J}$.

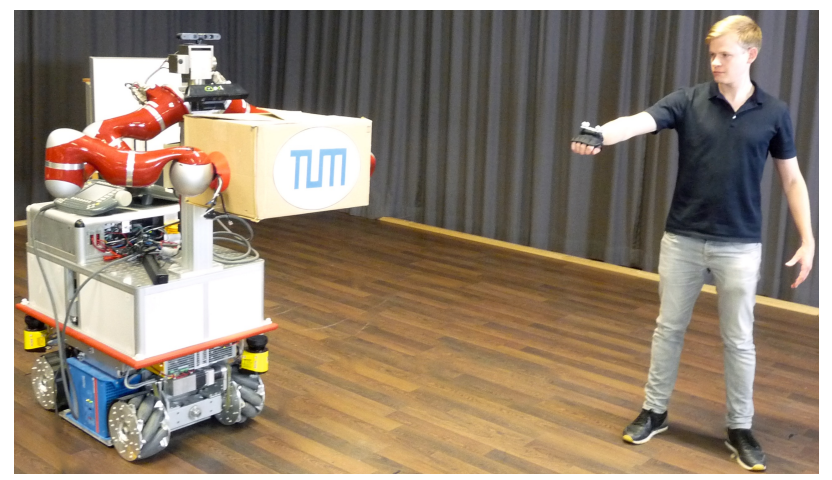

Fig. 5. Experimental figure. The human and the robotic system share the workspace. The human teleoperates the robotic system by the hand motion.

operative manipulation task. We used the port-Hamiltonian framework to model the constrained robotic system and showed that the representation of the system on the constrained manifold is again a port-Hamiltonian system. The proposed control approach maintains the desired formation between the robots and the desired behavior of the overall system, commanded by the human. We show that the interconnection of the proposed controller and the model is a port-Hamiltonian system. The complete system is passive and allows for a safe interaction with the human. The control approach is successfully validated in the simulation and the experiments. In the future we plan to extend the analysis to the interaction with the environment. 


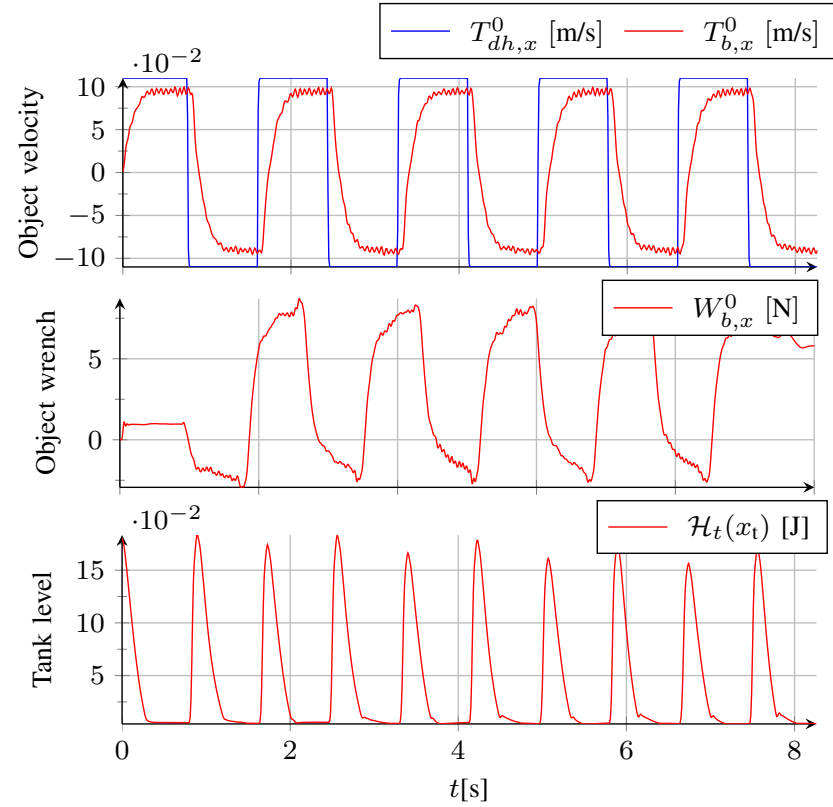

Fig. 6. Experimental results. Top: commanded and actual object velocity. Middle: force applied on the object. Bottom: energy level of the tank with $\mathcal{H}_{t}^{\max }=0.2 \mathrm{~J}$ and $\mathcal{H}_{t}^{t h}=0.01 \mathrm{~J}$.

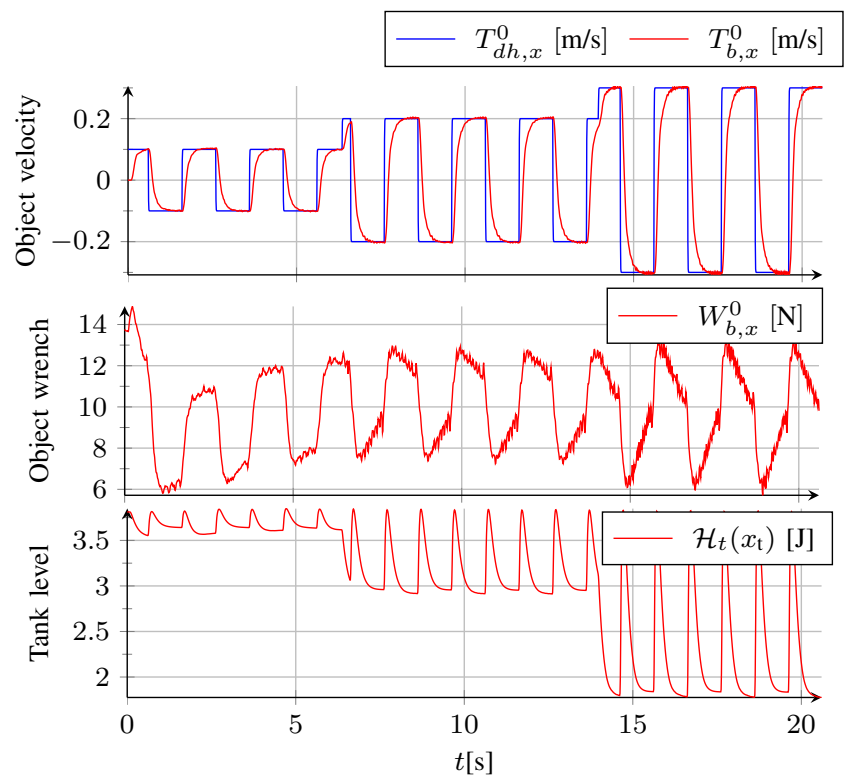

Fig. 7. Experimental results. Top: commanded and actual object velocity. Middle: force applied on the object. Bottom: energy level of the tank with $\mathcal{H}_{t}^{\max }=3.8 \mathrm{~J}$ and $\mathcal{H}_{t}^{t h}=1 \mathrm{~J}$.

\section{REFERENCES}

[1] D. Lee and S. M.W., "Bilateral teleoperation of multiple cooperative robots over delayed communication networks: Theory," in IEEE Int. Conf. on Robotics and Automation, 2005.

[2] G. Antonelli and S. Chiaverini, "Kinematic control of platoons of autonomous vehicles," IEEE Trans. Robot., vol. 22, no. 6, pp. 1285-1292, 2006.

[3] A. J. van der Schaft, "Port-Hamiltonian systems: an introductory survey," in Proc. Int. Congress of Mathematicians, 2006, pp. 1339-1365.

[4] M. Franken, S. Stramigioli, S. Misra, C. Secchi, and A. Macchelli, "Bilateral Telemanipulation With Time Delays: A Two-Layer Approach Combining Passivity and Transparency," IEEE Trans. Robot., vol. 27, no. 4 , pp. 741-756, 2011

[5] M. Geravand, E. Shahriari, A. De Luca, and A. Peer, "Port-based
Modeling of Human-Robot Collaboration towards Safety-enhancing Energy Shaping Control," in Proc. IEEE Int. Conf. on Robotics and Automation, May 2016, pp. 3075-3082.

[6] S. Stramigioli, B. Maschke, and A. van der Schaft, "Passive output feedback and port interconnection," in Proc. 4th IFAC Symp. Nonlinear Control Systems, 1998, pp. 613-618.

[7] E. Vos, J. M. Scherpen, A. J. van der Schaft, and A. Postma, "Formation control of wheeled robots in the port-Hamiltonian framework," in Proc. 19th IFAC World Congress, 2014, pp. 6662-6667.

[8] R. Ortega, A. J. van der Schaft, B. Maschke, and G. Escobar, "Energyshaping of port-controlled Hamiltonian systems by interconnection," in Proc. IEEE Conf. on Decision and Control, vol. 2, 1999, pp. 1646-1651 vol.2.

[9] R. Ortega, A. van der Schaft, F. Castanos, and A. Astolfi, "Control by interconnection and standard passivity-based control of porthamiltonian systems," IEEE Trans. Autom. Control, vol. 53, no. 11, pp. 2527-2542, 2008.

[10] A. Franchi, C. Secchi, H. I. Son, H. H. Bulthoff, and P. R. Giordano, "Bilateral teleoperation of groups of mobile robots with time-varying topology," IEEE Trans. Robot., vol. 28, no. 5, pp. 1019-1033, Oct 2012.

[11] M. Laffranchi, N. G. Tsagarakis, and D. G. Caldwell, "Safe human robot interaction via energy regulation control," in Proc. IEEE/RSJ Int. Conf. on Intelligent Robots and Systems, Oct 2009, pp. 35-41.

[12] T. S. Tadele, T. J. A. de Vries, and S. Stramigioli, "Combining energy and power based safety metrics in controller design for domestic robots," in Proc. IEEE Int. Conf. on Robotics and Automation, May 2014, pp. 1209-1214.

[13] S. Stramigioli, Modeling and IPC Control of Interactive Mechanical Systems: A Coordinate-Free Approach, ser. LNCIS. Springer, 2001, vol. 266.

[14] S. Erhart and S. Hirche, "Model and analysis of the interaction dynamics in cooperative manipulation tasks," IEEE Trans. Robot., vol. 32 , no. 3, pp. 672-683, 2016.

[15] A. J. van der Schaft, "Port-Hamiltonian Differential-Algebraic Systems," in Surveys in Differential-Algebraic Equations I, A. Ilchmann and T. Reis, Eds. Springer, 2013, pp. 173-226.

[16] S. Stramigioli, C. Melchiorri, and S. Andreotti, "A passivity-based control scheme for robotic grasping and manipulation," in Proc. IEEE Conf. on Decision and Control, 1999, pp. 2951-2956.

[17] S. Haddadin, Towards Safe Robots - Approaching Asimov's 1st Law, ser. STAR. Springer, 2014, vol. 90.

[18] J. L. Wood, "Dynamic response of human cranial bone," J. Biomechanics, vol. 4, no. 1, pp. 1 - 12, 1971.

[19] N. Yoganandan, F. Pintar, D. Maiman, J. Cusick, A. Sances, and P. Walsh, "Human head-neck biomechanics under axial tension," Medical Engineering and Physics, vol. 18, no. 4, pp. 289 - 294, June 1996.

[20] S. Stramigioli, R. Mahony, and P. Corke, "A novel approach to haptic tele-operation of aerial robot vehicles," in Proc. IEEE Int. Conf. on Robotics and Automation, May 2010, pp. 5302-5308. 